\title{
Processing of array sonic logging data with multi-scale STC technique
}

\author{
Tao Guo *, He Fengjiang, Yue Wenzheng and Chen Ping
}

CNPC Well Logging Key Laboratory, China University of Petroleum, Beijing 102249, China

\begin{abstract}
It is desirable to develop new signal processing techniques for effectively extracting reflected waves under the strong interferences of borehole guided waves. We presented a multi-scale semblance method for the separation and velocity (slowness) analysis of the reflected waves and guided waves in borehole acoustic logging. It was specially designed for the newly developed tools with ultra-long sourcereceiver spacing for acoustic reflection survey. This new method was a combination of the dual tree complex wavelets transform (DT-CWT) and the slowness travel time coherence (STC) method. Applications to the 3D finite difference (FD) modeling simulated data and to the field array sonic waveform signals have demonstrated the ability of this method to appropriately extract the reflected waves under severe interference from the guided waves and to suppress noise in the time-frequency domain.
\end{abstract}

Key words: Borehole acoustic logging, signal processing, wavelet transform, reflection survey

\section{Introduction}

Modern acoustic logging tools can also be used to detect the waves reflected from the near-borehole structural features, in addition to the borehole guided waves for formation elastic velocity analysis. These reflected waves can be used to form an image of the near-borehole structural features (Tang, 2004). Possible applications include horizontal well logging, structural dip and contour determination, fracture zone imaging and enhanced oil recovery (EOR) steam-flood monitoring. Because the reflected waves from near-borehole geological structures are usually mixed with the dominating borehole guided waves, the most common methods for velocity (slowness) analysis of array sonic logging data, such as the STC method based on the statistic analysis of signal energy, are hampered. It is desirable to develop new signal processing techniques for effectively extracting reflected waves under the strong interferences of borehole guided waves for the new logging tools.

In recent years, many wavelet processing methods with multi-scale analysis have been developed and introduced into seismic and well logging data-processing mainly for coping with non-stationary noise (Miller et al, 2004; He et al, 2005; Yue and Tao, 2003; Yue et al, 2006; Tao et al, 2006). Despite demonstrated remarkable abilities in recovering very weak signals, those methods can not be applied to the data processing of acoustic array measurements directly as they are not shift invariant. To achieve the shift invariance while preserving the other advantages of these multi-scale analysis algorithms, we introduce in this paper the DT-CWT developed by Kingsbury (2003) into the wavelet processing.

*Corresponding author. email: taoguo@vip.sina.com Received May 1, 2008
The key for the DT-CWT is designing the Q-shift orthogonal filter properly to ensure the required properties of avoidance of aliasing, perfect reconstruction and orthogonality. By combining the DT-CWT with the STC, we constructed a multi-scale semblance method for the signal processing of the reflected and guided waves. In this way, we could get STC information at different scales to eliminate the non-stationary interference noise more efficiently. Approximate shift invariance, good directional selectivity and computational efficiency of such a DT-CWT and STC combination made it a suitable algorithm for the data processing of the reflected wave logging method. We subsequently applied this method to the 3D FD modeling simulated data and to the field array sonic waveform signals from the newly developed tool. Our results have demonstrated that this method can appropriately extract the reflected waves from very strong interferences of the guided waves while effectively suppressing all noise in the time-frequency domain.

\section{Principle and algorithm of the multi-scale semblance method}

The shift invariance in wavelet analysis means that the wavelet coefficients would not change appreciably with a slight shift of the input signal in time domain (Fernandes et al, 2005). The major factor that causes the usual discrete wavelet transform (DWT) to be not shift invariant lies in its down sampling. The DT-CWT was developed and introduced by Kinsbury (2003) in order to achieve significant improvements over the usual DWT for approximate shift invariance. He pointed out that the key to obtaining shift invariance from the dual tree structure lies in designing the filter delays at each stage, so that the low-pass filter outputs in tree $b$ are sampled at points midway between the sampling points of 
the equivalent filters in tree $a$. This requires a delay difference between $a$ and $b$ low-pass filters of one sample period at tree level 1, and of half sample period at subsequent levels. Selesnick $(2001 ; 2004)$ showed that these low-pass delay constraints produced a Hilbert pair relationship between the wavelet bases for the two trees. This leads naturally to the interpretation of the outputs from trees $a$ and $b$ as the real and imaginary parts respectively of complex wavelet coefficients. For levels 2 and below, we designed a Q-shift filter following the algorithm developed by Kinsbury (2003). Considering the features of array sonic signals and the requirements for highly smooth decomposition of various noise and Stoneley wave etc. into different scales, in this study we took the biorthogonal wavelet bior 13.19 as the first level filter and set the length of the Q-shift filter as 48 taps $(n=24)$ with a cutoff at $\pi / 3$.

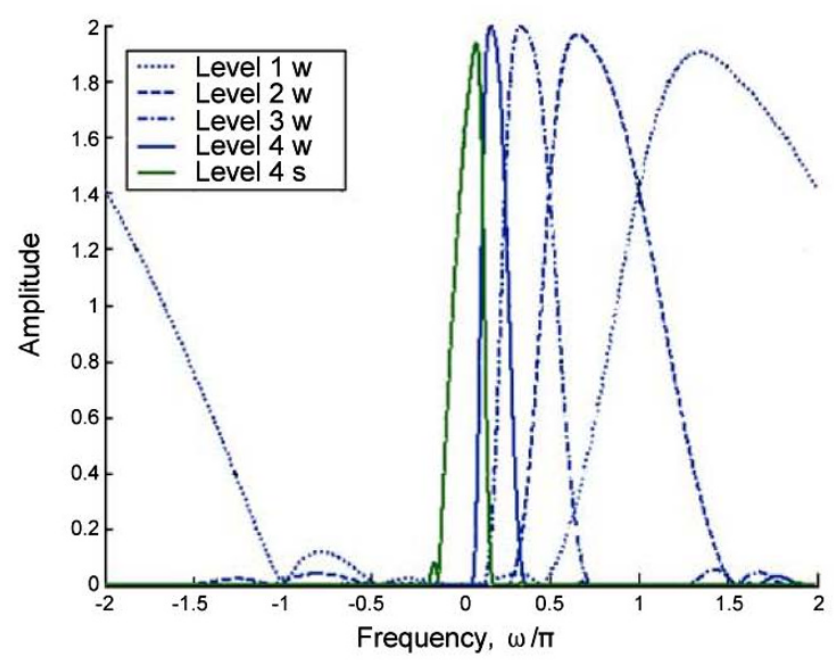

Fig. 1 Frequency responses of the optimized complex filter at each scale

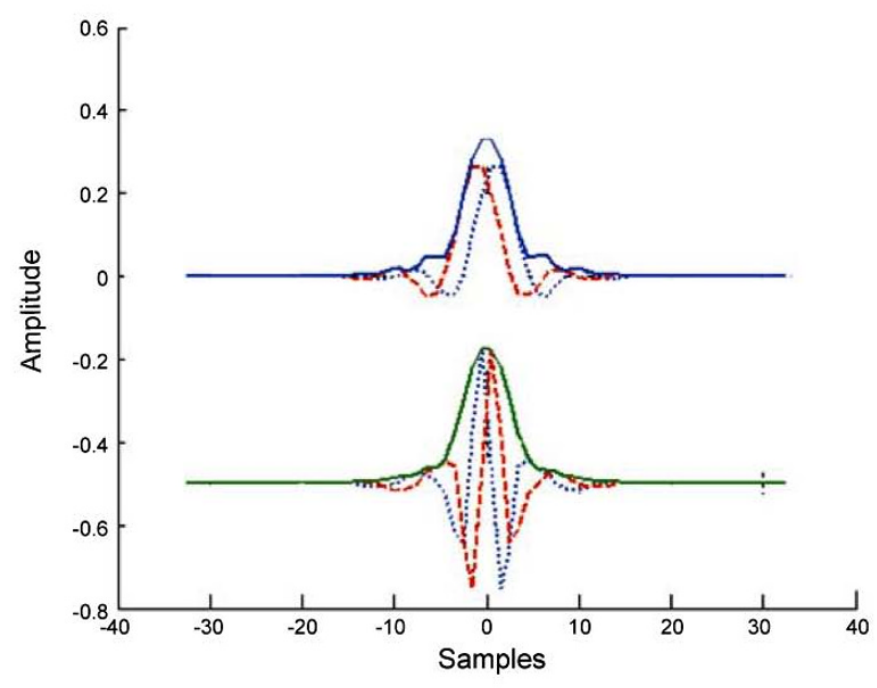

Fig. 2 Impulse responses of the filter band at level 2. Wavelet (blue) response is on the top and scaling function (green) is below

Fig. 1 shows the frequency responses of the optimized complex filter at each scale. In this figure, w and s represent wavelets (band-pass filter) and scaling (low-pass filter) functions, respectively. It can be seen from this figure that the negative frequencies are effectively cancelled and the positive frequencies are enhanced.

Fig. 2 shows the impulse responses of the filter band at level 2 , where the wavelet is on the top and scaling function is below; the dashed curves (red) and the dotted curves (blue) are for tree $a$ and tree $b$, respectively. The solid curves show the magnitudes of the complex waveforms.

There have been many methods for computing the slowness from the waveforms of array sonic logging. The most common one is the so-called STC method. A typical STC algorithm consists of the following three steps: 1. A set of time windows is applied to the waveforms, with the window positions determined by an assumed arrival time at the first receiver and an assumed slowness. For a range of values of arrival time and slowness, the scalar semblance is computed for the windowed waveform segments; 2 . Identify the local maximum of the semblance function with a peakfinding algorithm; 3 . Label the peaks in the semblance contour plot for each wave mode based on the criterion of the physical constraints and compute the corresponding slowness and travel time, and plot these values on a graph whose axes are slowness and depth. The major problem with the STC algorithm for this case is that it is prone to the error in the presence of much stronger borehole guided modes and various noise including the random noise of higher frequency and the measuring device noise of lower frequency. These interferences degrade the correlation of weak signals and hence cause the difficulties for labelling peaks correctly. Though applying a band-pass filter in frequency domain could remove part of this noise, it would change the shape of the waveforms (Kimball and Marzetta, 1984; Kimball, 1998) and degrade the correlations of the desired signals. Wavelet analysis has been proven to be able to effectively separate the desired signals from noise by processing the array sonic waveforms in time-scale (frequency) domain. The algorithm of multi-scale STC is the combination of DT-CWT and STC to achieve both the effective denoising and the robustness in processing array sonic measurements. This algorithm can be outlined as following procedures:

1) Apply DT-CWT transform to every waveform trace in the common shot point gathers (CSP) naturally recorded by the tool;

2) Form common scale gathers by sorting all the wavelet coefficients in a scale from every trace;

3) Perform inverse transform on these common scale gathers after they are denoised through a soft threshold scheme (Mou, 1986);

4) Compute STC for every reconstructed common scale gather to obtain STC contour plots for different scales.

\section{Results for processing synthetic and field data of acoustic reflection survey}

We first applied this multi-scale STC algorithm to the waveforms from finite difference (FD) modeling to extract the weak reflection signals. Fig. 3 shows the full waveforms of the acoustic reflection logging data by FD modeling. The spacing between the source and the nearest receiver is set at 
$8 \mathrm{~m}$ and the interval between receivers is $7.5 \mathrm{~cm}$. Because the amplitudes of compressional head waves and reflected waves are very small, it is hard to see them directly from this figure. Fig. 4(a) and Fig. 4(b) demonstrate the resulted common scale gathers at the first scale and at the third scale respectively. Fig. 4(c) and Fig. 4(d) show the corresponding STC contour plots as examples. It can be understood that the first scale gather contains mainly the low frequency noise with little correlation, distributed in Pseudo-Rayleigh and Stoneley bands and the reflected events are distributed in other scale gathers, mainly the third scale gather. Only the third scale gather is shown here. But the reflected wave is still very difficult to be identified in this gather as the Pseudo-Rayleigh energy is still dominating in this band.

Comparing the corresponding STC contour plots, the P-P reflected wave is clearly prominent on the third scale STC contour plot positioned at the slowness of $60 \mu \mathrm{s} / \mathrm{ft}$ and the travel time of $3700-4700 \mu \mathrm{s}$. Appearing clearly also are the compressional head waves at the slowness of $101 \mu$ s and the Pseudo-Rayleigh event at the slowness of $184 \mu$ s with moderate dispersion. These features are in very good agreement with the original model for FD modeling simulations. These STC plots (2nd and 4th scale STC plots are similar but not shown here) also demonstrate the shift invariance of the DTCWT (Kinsbury, 2003; Selesnick et al, 2005; Ng et al, 2006).

We subsequently applied this new method to the array sonic waveforms of field monopole logs. The most significant

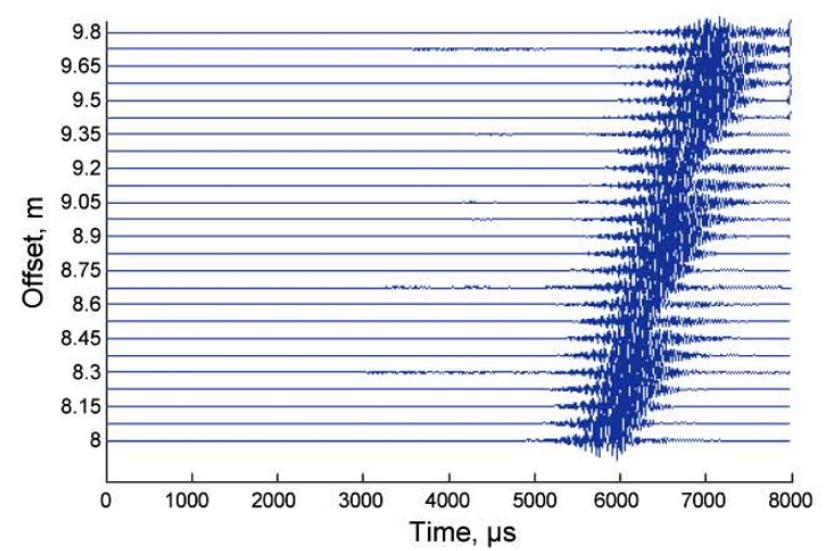

(a)

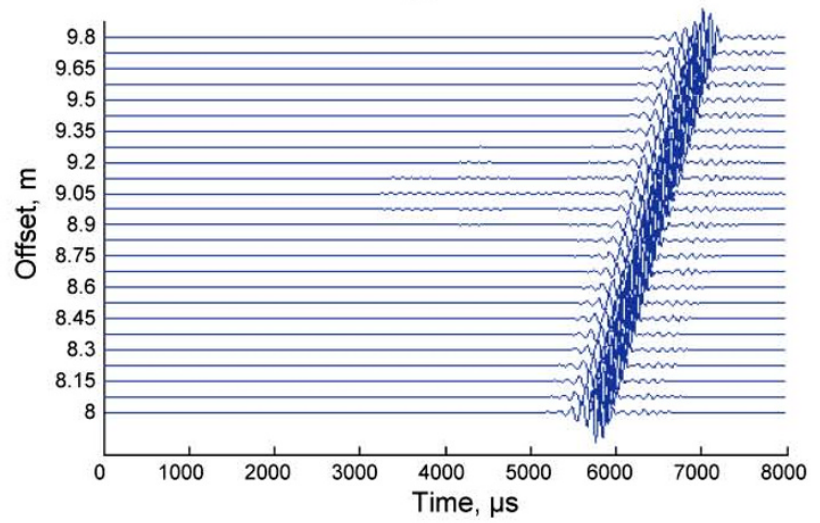

(b) difference between the numerical modeling data and the field data lies in their noise levels. The fact that the real field data are usually contaminated by various noise makes it the first priority for all processing algorithms to be able to effectively denoise. Fig. 5(a) and Fig. 5(b) show a set of array sonic waveforms from field monopole measurements and the reconstructed waveforms after we applied the multi-scale STC denoising operations. It can be seen that both the higher and lower frequency noise are effectively removed.

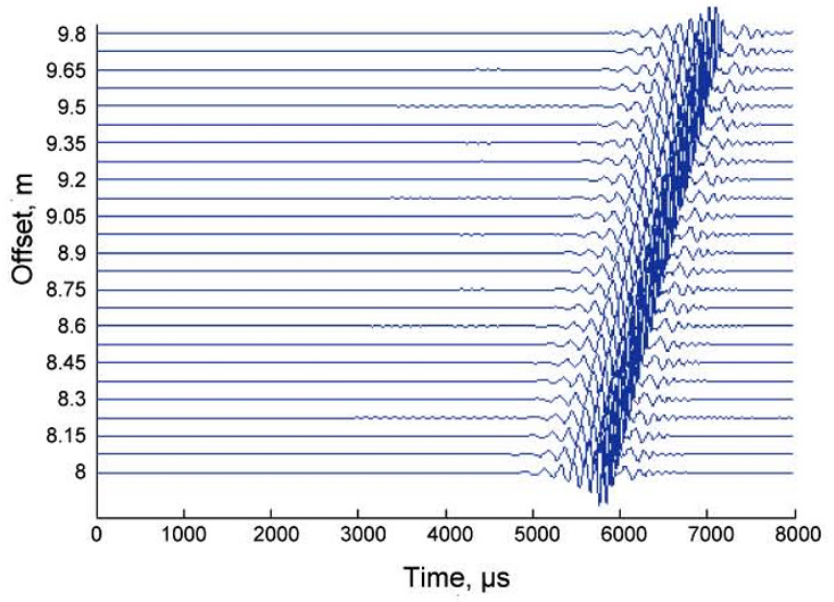

Fig. 3 Full waveform array sonic logging data by FD

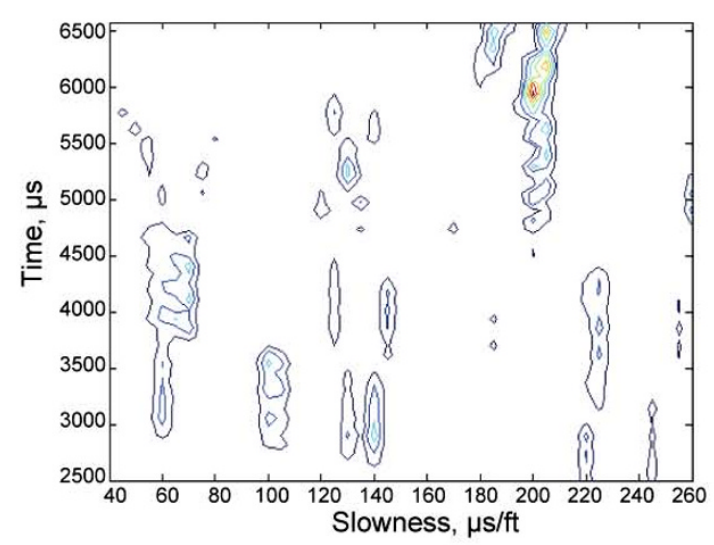

(c)

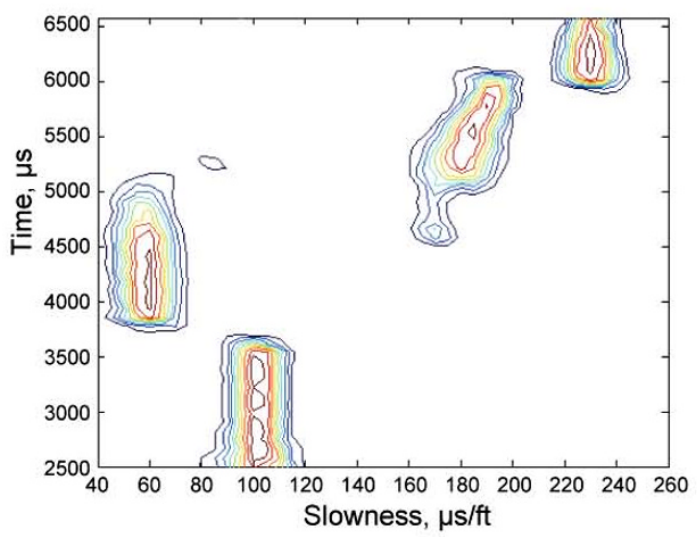

(d)

Fig. 4 Common scale gathers at the first (a) and the third (b) scales and the corresponding STC contour plots (c and d) 


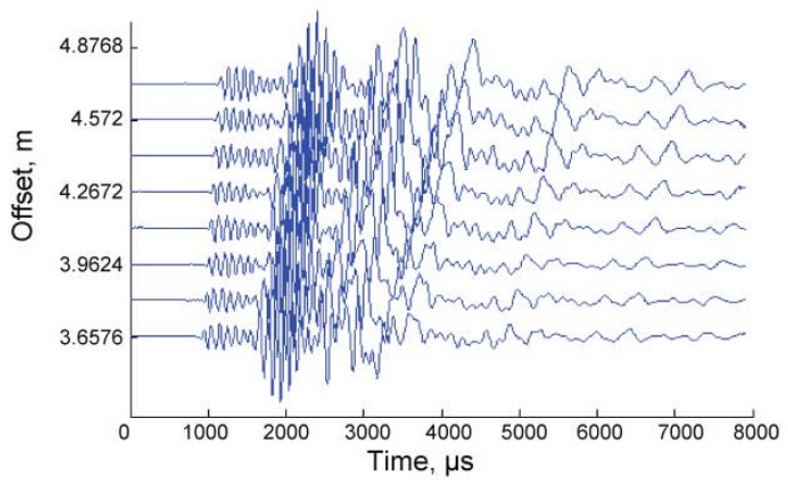

(a)

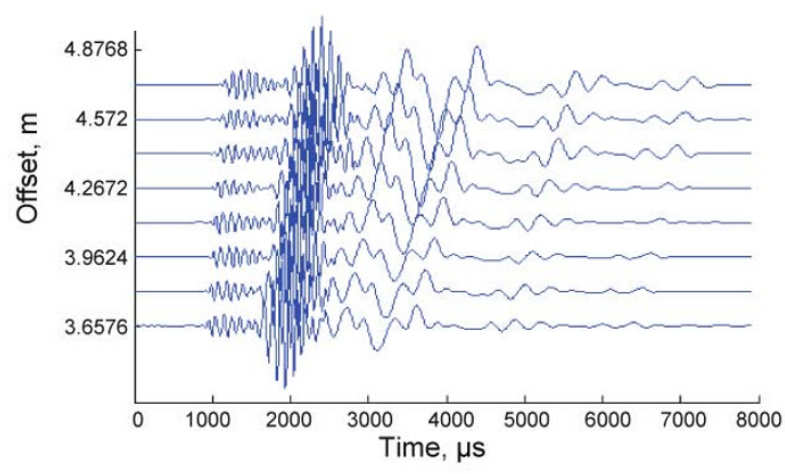

(b)

\section{Conclusions}

We developed a multi-scale semblance method for the separation and velocity (slowness) analysis of the reflected waves and guided waves in borehole acoustic logging. This new method was a combination of the DT-CWT and the STC algorithms. The essence of the DT-CWT was designing the Q-shift orthogonal filter properly to ensure the required properties of aliasing avoidance, perfect reconstruction and orthogonality. Applications of this new method to the simulated FD modeling acoustic reflection survey data and to the field array sonic waveform signals have demonstrated the effectiveness of this method to appropriately extract the reflected waves from strong interferences of the guided waves and suppress the noise in all time-frequency domain.

\section{Acknowledgements}

This work is supported by National Natural Science Foundation of China (the project No. is 50674098), the National 863 Project of China (Grant 2006AA06Z207), the National Basic Research Program of China (973 Program, 2007CB209601).

\section{References}

Fernandes F C A, Wakin M B and Baraniuk R G. Non-redundant, linearphase, semi-orthogonal, directional complex wavelets. Proceedings of IEEE International Conference Acoustics, Speech and Signal Processing (ICASSP). Montreal, May 2004. (2): 953-956

He F J, Tao G and Luo H Y. SNR estimation in NMR well logging by wavelet analysis. Nuclear Electronics and Detection Technology. 2005. (5): 471-474 (in Chinese)

Kimball C V and Marzetta T L. Semblance processing of borehole acoustic array data. Geophysics. 1984. 49(3): 274-281

Kimball C V. Shear slowness measurement by dispersive processing of the borehole flexural mode. Geophysics. 1998. 63(2): 337-344

Kingsbury N. Design of Q-shift complex wavelets for image processing using frequency domain energy minimization. Proceedings of 2003 International Conference on Image Processing. 2003. (1): 1013-1016

Miller M, Kingsbury N and Hobbs R. Seismic imaging using complex wavelets. Proceedings of IEEE International Conference on Acoustics, Speech and Signal Processing (ICASSP). Philadelphia, March 2005. (2): 557-560

Mou Y G. Data processing methods in seismic exploration. Beijing: Petroleum Industry Press. 1986. 49-51 (in Chinese)

Ng J, Prager R, Kingsbury N, et al. Modeling ultrasound imaging as a linear, shift-variant system. IEEE Transactions on Ultrasonics, Ferroelectrics and Frequency Control. 2006. 53(3): 549-563

Selesnick I W. Hilbert transform pairs of wavelet bases. IEEE Signal Processing Letters. 2001. 8(6): 170-173

Selesnick I W. The double-density dual-tree discrete wavelet transform. IEEE Transactions on Signal Processing. May 2004. 52(5): $1304-1314$

Selesnick I W, Baraniuk R G and Kingsbury N C. The dual-tree complex wavelet transform. IEEE Signal Processing Magazine. November 2005. 22(6): 123-151

Tang X M. Imaging near-borehole structure using directional acousticwave measurement. Geophysics. 2004. 69(6): 1378-1386

Tao G, He F J, Wang B, et al. Multi-scale analysis for azimuthal anisotropy of reservoir rocks from cross-dipole logs. Journal of Geophysics and Engineering. 2006. (2): 135-142

Yue W Z and Tao G. Applications of wavelet transform in reservoir fluid typing. Chinese Journal of Geophysics. 2003. 46(6): 863-869 (in Chinese)

Yue W Z, Tao G and Liu Z W. Identifying reservoir fluids by wavelet transform of well logs. SPE Reservoir Evaluation and Engineering. 2006. 9(5): 524-530

(Edited by Hao Jie) 